\title{
Modelo de optimización de la cadena de distribución de la agricultura familiar campesina en las parroquias Quingeo y Santa Ana del Cantón Cuenca
}

Distribution chain optimization model of peasant family farming in the Quingeo and Santa Ana parishes of Cuenca Canton

María Cecilia Piedra Rivas. ${ }^{1}$, Christian Mauricio Banegas Campoverde. ${ }^{2}$ \& Yonimiler Castillo Ortega. ${ }^{3}$

\begin{abstract}
.
DOI: https://doi.org/10.33262/concienciadigital.v4i2.1630

Effective comercialization, as well as the direct delivery of products to the consumer, by farming families, requires an effective knowledge of the distribution chains dynamics. Traditionally, this is obtained by improving value chains and access to markets, however, there are other critical factors that are involved in the process and that also can interact differently in each community. The purpose of this study was to establish a model for the distribution chain optimization of the of peasant family farming in Quingeo and Santa Ana parishes. The methodology was based on the preparation of surveys to diagnose the main obstacles along the route of peasant family farming. This study shows that the most serious problems of farmers are the lack of market, transportation, unfair prices and diffusion. Second, organizational strengthening and technical assistance also appear as important problems. The study made it possible to define a distribution chain optimization model that visualizes the complexity of the process.

\footnotetext{
${ }^{1}$ Universidad Católica de Cuenca, Maestría en Desarrollo Local Mención en Ordenamiento Territorial, Azuay, Ecuador, cecilia.piedra@yahoo.es https://orcid.org/0000-0003-2813-2745.

2 Universidad Católica de Cuenca, Carrera de Economía, Azuay, Ecuador, cbanegas@ucacue.edu.ec https://orcid.org/0000-0003-1102-2283

3 Universidad Católica de Cuenca, Carrera de Economía, Azuay, Ecuador, ycastilloo@ucacue.edu.ec https://orcid.org/0000-0002-7710-5199
} 
Keywords: Peasant family farming, Distribution Chains, Local Economic

Development, markets, commercialization, correspondence analysis

\section{Resumen.}

Introducción. La comercialización efectiva, así como la entrega directa de productos hacia el consumidor, por parte de familias de agricultores, requiere de un conocimiento eficaz de la dinámica de las cadenas de distribución. Tradicionalmente esto se obtiene mediante la mejora de las cadenas de valor y el acceso a los mercados, sin embargo, existen otros factores críticos que están involucrados en el proceso y que además pueden interactuar de manera distinta en cada comunidad. Objetivo Establecer un modelo de optimización de la cadena de distribución de la agricultura familiar campesina de las parroquias Quingeo y Santa Ana. Metodología se basó en la elaboración de encuestas para diagnosticar los principales obstáculos a lo largo de las rutas de la agricultura familiar campesina. Resultados Este estudio muestra que los problemas más graves que tienen los agricultores son la falta de mercado, transporte, precios injustos y difusión. En segundo lugar, aparecen también como problemas importantes el fortalecimiento organizativo y asistencia técnica. Conclusión. Se concluyó que el estudio permitió definir un modelo de optimización de la cadena de distribución que visualiza la complejidad del proceso.

Palabras claves: Agricultura familiar campesina, cadenas de distribución, desarrollo económico local, mercados, comercialización, análisis de correspondencias

\section{Introducción.}

Según la Fundación Heifer Internacional (2018), se define a la agricultura familiar campesina como una producción estrechamente vinculada a la mano de obra de los miembros de la familia. Esta producción implica determinar las decisiones de la familia en cuanto a la elección de las actividades productivas, el modelo de gestión de los recursos, la organización del trabajo, la transmisión de los saberes y el patrimonio, el grado de articulación al mercado, entre otros.

De acuerdo con Ayora (2016), el sector campesino se encuentra debilitado a nivel del país. A nivel mundial en el año 1950 el $30 \%$ de la población radicaba en las ciudades, y para el año 2000 aproximadamente el $47 \%$ habitaban en las zonas urbanas. Se tiene previsto que para el 2030 la cifra alcanzaría el 60\%. Ante esta realidad, hay diferentes teorías que han abordado sobre "el fin del campesinado" y la "metamorfosis campesina" y se menciona que su desaparición sería irreversible. De hecho, esta situación ya fue una realidad en China algunos países de Europa. En latinoamericana, Brasil fue el escenario de un éxodo rural entre los años 1960 y 1980 con alrededor de 27 millones de desplazados. 
Al respecto, Maletta (2016) indica que en el Ecuador existe un predominio de la agricultura empresarial sobre la familiar, motivo por el cual su permanencia

se ha convertido en un reto, puesto que su pilar fundamental es la familia y sus relaciones con las comunidades. Para entender su esencia es necesario mencionar la historia de su concepto, el cual surge con las concepciones utópicas y populistas presentes en el siglo XIX y comienzos del siglo XX. Su primera característica es la de una finca familiar la cual no requería de mano de obra asalariada. Es necesario acotar que las fincas agrícolas están presentes en la economía capitalista como es el caso de América Latina en donde se acepta la contratación de personal. Ante este escenario se debe señalar que este tipo de agricultura tiende a disgregarse o a desaparecer, otras se convierten en empresas capitalistas (pequeñas, medianas o grandes), otras por el contrario suelen organizarse en cooperativas, sin embargo, otras, aunque muy pocas persisten como fincas exclusivamente trabajadas por una familia.

El sector agrícola en el Ecuador se ha desarrollado de forma incipiente y a pesar de las muchas ofertas de gobiernos de una revolución agraria esto sólo ha quedado en la utopía, puesto a que se ha dado mayor énfasis a actividades extractivistas tales como la industria petrolera, evidenciando nuestra posición como país primario exportador. El sector agropecuario aporta a la economía ecuatoriana con el $47 \%$, ubicándose después del petróleo y minería. Además, cubre el $95 \%$ de la demanda interna de los alimentos que consume la población, así como también genera empleo al $25 \%$ de la población económicamente activa (PEA) (Pino, Hermes, Apolo, \& Sisalema, 2018).

La agricultura familiar campesina en el país, se enfrenta a un escenario complejo, puesto que según Peñafiel (2017), el último censo agropecuario aplicado en Ecuador en el año 2000 dio a conocer que se privilegia a la Agricultura Empresarial. Este tipo de agricultura se caracteriza por el monocultivo y la concentración del $80 \%$ de la producción, y del 15 $\%$ de las UPAS (Unidad de Producción Agropecuaria), además utiliza cerca del $63 \%$ del agua para riego.

La normativa nacional presente en el Plan Nacional de Desarrollo (antes Plan Nacional del Buen Vivir) aborda nueve objetivos nacionales a partir de tres ejes, en los cuales se reconoce que la economía debe estar al servicio de la comunidad, la garantía de derechos a lo largo del ciclo de vida y la participación de la sociedad y la gestión estatal para el cumplimiento de objetivos nacionales (Secretaría Nacional de Planificación y Desarrollo, 2017). Estos objetivos se consideran como bases esenciales para la sustentabilidad ambiental y el desarrollo territorial equitativo.

Ante este escenario, la Organización de las Naciones Unidas para la Alimentación y la Agricultura (2014) declaró al 2014 como el "Año Internacional de la Agricultura Familiar" (AIAF) con la finalidad de crear conciencia en los gobiernos, sobre la importancia de este tipo de producción, puesto que aproximadamente el $70 \%$ de la alimentación diaria proviene de este tipo de producción denominada de subsistencia. 
El Ministerio de Agricultura en el año 2011 aplicó la idea de impulsar estrategias de comercialización alternativa de productos provenientes de los pequeños y medianos productores de la agricultura familiar campesina, denominados Circuitos Alternativos de Comercialización (CIALCOS), (Barrionuevo et al., 2018).

En el marco de la crisis que vive la Economía Ecuatoriana, para las zonas rurales las estrategias implementadas para salir de la pobreza y mejorar la calidad de vida, consisten en desarrollar sus propias habilidades de desarrollo local. Aquí se incluye el involucramiento de los actores que se encuentran en un determinado lugar, con la finalidad de crear acciones que permitan tener acceso a sus propios bienes y servicios y de esta manera contribuir a un desarrollo equilibrado y sostenible (Orellana, 2001).

\section{Aspectos teóricos sobre las tendencias del desarrollo económico local}

$\mathrm{Al}$ analizar las diferentes ideas teóricas de desarrollo económico local, está claro que es imprescindible para el crecimiento de los pueblos que se desarrollen las capacidades locales mediante capacitaciones (Naciones Unidas, 2015). De esto se desprende que las organizaciones deben cuidar su capacidad de trabajar con diversos de actores para garantizar la diversificación de fuentes de financiamiento. Los intercambios a través de la creación de redes y el esfuerzo por gestionar el conocimiento también son factores de gran utilidad. Estas son prácticas ampliamente apoyadas y parecen ser un mecanismo esencial para mejorar el conjunto de habilidades y el conocimiento agregado (el saber hacer) de las agencias de desarrollo (Clark, G. Huxley, J., \& Mountford, D. 2012).

De acuerdo con Bingham y Mier (1993), se considera que el desarrollo económico local es una fuente de creación de riqueza, donde se mejora la economía de las familias mediante la creación de empleo. Esto conlleva al sector privado a crear dicha riqueza mediante empleos, produciendo bienes y servicios y realizando intercambios. El mismo autor considera que el sector público facilita y promueve la creación de empleos y riqueza del sector privado y asegura que en el corto y largo plazo se sirva a los intereses de la mayoría de la población. En este sentido el desarrollo económico local, es imprescindible para el desarrollo de los pueblos, donde es importante que se involucren las bases con capacitación, creación de capacidades locales e intercambio de experiencias para el éxito de los proyectos de desarrollo, especialmente los que la visión de dar valor agregado a los productos para su comercialización.

De acuerdo al análisis de Pérez (2004), se establece que las causas del comienzo de la crisis económica en los países desarrollados a finales de los años setenta, tuvieron un impacto negativo debido el deterioro de las condiciones económicas en algunos territorios. Ante ello, las medidas diseñadas por los gobiernos centrales resultaron ineficientes ya que impedían incorporar acciones en los sistemas productivos locales. Además, el creciente proceso de transnacionalización, globalización y apertura externa de las economías, ha ido mostrando de forma cada vez más clara, la importancia de la 
heterogeneidad y la mayor exposición de los diferentes sistemas productivos locales ante las exigencias de la actual fase de transición y cambio del paradigma tecno económico. Vázquez (2000) sostiene que el desarrollo económico local es un logro en la economía de las visiones territoriales, donde se consideran tres aspectos: el económico, socio cultural y política administrativa. El primero se refiere a la facilidad que tienen los empresarios locales, a ser eficientes en los factores productivos, generando grandes economías de escala para el aumento de la productividad, consiguiendo así ser competitivos en el mercado. En el segundo aspecto se involucran las relaciones económicas y sociales, en donde las instituciones son la base fundamental de apoyo en el proceso de desarrollo. En el tercero se involucran los trabajos locales que crean un entorno favorable a la producción e impulsan el desarrollo. Con esto nos damos cuenta que son procesos muy importantes para poder llevar adelante la economía de los pueblos y poder trabajar en las cadenas de distribución agroalimentarias.

\section{Cadenas de Distribución Agroalimentarias}

El estudio de las cadenas de distribución ha sido y es actualmente un tema de vital importancia para los productores de la agricultura familiar campesina, ya que deben enfrentarse a varios desafíos como son falta de mercados, monopolio, falta de transporte, asistencia técnica, precios injustos, entre otros. En este sentido, lo que se pretende es buscar un mecanismo que les sirva de apoyo con estos inconvenientes, y dejar plasmado un modelo de distribución que permita al agricultor mejorar sus ventas y por ende dinamizar la economía asegurando su alimentación y la calidad de vida de los pequeños y medianos agricultores.

Las cadenas de valor permiten describir las actividades realizadas en un determinado sector, con el propósito de generar valor agregado para la venta hacia el consumidor final. Incluso analizan que con este sistema el producto llegue fresco y de buena calidad hacia el consumidor. Además, son capaces de integrar sistemas de información, producción y distribución de los productos. Para ello vieron conveniente adaptar un esquema de la cadena de valor y agentes económicos representativos. Porter (1986) y Díaz, Campos y Gómez, (2012) consideran al agricultor como el punto clave en la cadena valor y distribución, para ello ven conveniente crear cuatro etapas; producción, transformación, distribución política y distribución minorista. La primera genera la materia prima, sin medir la transformación, esta etapa afecta especialmente en la competitividad al rato de hacer la negociación con los compradores. La segunda se basa cuando se da valor agregado a los productos. La tercera consiste en la distribución de logística, en donde se involucran los mayoristas y las plataformas de distribución que incluyen las tareas de expediciones, tales como: transporte, recepción y gestión de pedidos. La cuarta etapa, que representa la distribución minorista, influye en la forma en la que los productos pueden ser entregados todos los días.

Cartier (2016) define a las "cadena Agroalimentarias" como procesos y actividades desarrolladas con el propósito de producir y distribuir alimentos para el consumo humano. 
Para ello se deben considerar recursos tales como: eslabón de producción primaria, eslabón industrial y productos de eslabón comercial.

Morelos Gómez, J. (2012) sostiene que: la integración de la cadena productiva tiene gran importancia en el flujo de los procesos de intercambio, materiales e información entre estos y su entorno que se encuentran dentro y fuera de los mercados, donde considera importante el involucramiento de los proveedores y clientes. Aquí se diferencian dos procesos: logístico y cadena de abastecimiento. El primero se basa en productos, servicios y aspectos financieros, donde se identifica la trayectoria desde la producción hasta el consumidor final. El segundo conlleva la gestión y el mejoramiento a lo largo de la cadena global. Estos elementos son la clave para mantener al cliente, y lograr el éxito de las cadenas de distribución agroalimentarias.

Referente al sector agroalimentario, Kirwan, J., Maye, D., \& Brunori, G. (2017), sostiene que en este sector se identifican varios elementos importantes como: volatilidad de precios de los productos alimenticios, una gran preocupación por la seguridad y soberanía alimentaria, los sistemas de gestión de energía empleados, y lo más importante crear conciencia sobre las amenazas causadas por el cambio climático.

Según Ahi, P., \& Searcy, C. (2013), la sostenibilidad puede alcanzar varias definiciones que se desarrollan con el pasar el tiempo, especialmente relacionándose con los aspectos ambientales. Esto tiene un enfoque basado en tres dimensiones: social, económica y ambiental. Con una base fundamentada desde el punto de vista de las organizaciones, se puede identificar la sostenibilidad del negocio, por otro lado, con el pasar del tiempo las organizaciones están articuladas estrechamente con sistemas ambientales, económicos y sociales, logrando de esta manera, manejar mejor los problemas internos y externos.

Analizando la sostenibilidad con respecto a lo empresarial, Reefke y Sundaram (2016), abordan la necesidad de manejar los temas económicos, social y ambiental de una manera integral y equilibrada.

Con respecto a la sostenibilidad del desarrollo de la cadena de valor alimentaria, según Neven (2015), el incremento de la sostenibilidad social y medioambiental, es el punto clave para las empresas agroalimentarias, debido a que determinan el ingreso a mercados competitivos, cumpliendo con las normas que rigen en cada localidad.

Uno de los problemas más graves de los productores de la agricultura familiar campesina (AFC), es que tienen menos posibilidades de crecer, ya que tienen escasas habilidades empresariales y además cuentan con nulos recursos para realizar campañas de publicidad y marketing. Es por ello que enfrentan una serie de amenazas, siendo una de ellas la falta de espacios adecuados para el expendio de sus productos, y además convirtiéndose en víctimas de los grandes monopolios de los mercados tanto mayoristas como minoristas. Por otro lado, los intermediarios son quienes fijan los precios que resultan poco ventajosos para el pequeño productor. Rebaï (2017) indica que la que la vulnerabilidad 
económica de los agricultores familiares de la provincia del Azuay, proviene principalmente de su falta de acceso al mercado debido a su innegable subordinación a intermediarios que limitan su integración comercial.

La cadena de valor agropecuaria, tiene un esquema bastante interesante, porque se puede evidenciar que hay una articulación estratégica de las alianzas comerciales, donde facilita al agricultor los canales de distribución con el propósito de tener éxito en las ventas tal como se puede observar en la Figura 1.

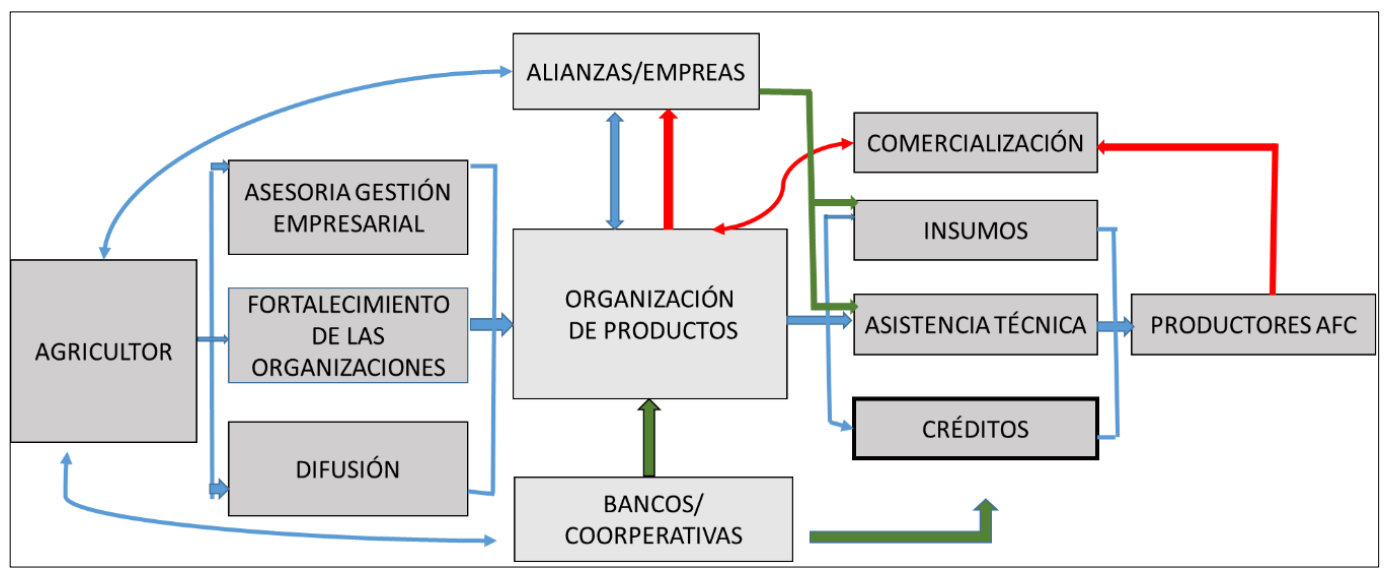

Figura 1: Canales de distribución de productos y articulación comercial. Fuente: Manrique, 2011

\section{Agricultura Familiar campesina}

La FAO propuso en el año 2008 la siguiente definición de la Agricultura Familiar: "La agricultura familiar es una forma de vida de organizar, la producción agrícola, pecuaria y silvícola, así como la pesca y la acuicultura, que es conducida por una familia y en su mayoría depende de mano de obra familiar". Tanto la familia como la explotación están vinculadas, co-evolucionan y se ajustan a funciones económicas, reproductivas, sociales, ambientales y culturales.

Según Lechón y Chicaiza (2019), la agricultura familiar requiere mejorar algunos aspectos tales como: las propuestas de distribución equitativa de la tierra, el acceso al mercado financiero, así como también el uso de semillas propias. Sin embargo, esto no se aplica en la realidad, ya que el principal obstáculo, para los agricultores es el mercado, quien está sujeto a las dinámicas donde prima la ley de la oferta y la demanda.

Por su parte, Peñafiel (2017) sostiene que una de las alternativas que aportan a la sostenibilidad de la producción familiar es disminuir la dependencia al mercado, dando paso a sistemas de auto dotación como lo es la agricultura familiar. De esta manera se podrá, además, recuperar la base de la alimentación de la población. Un ejemplo de ello son las cadenas de comercialización directa tales como las tiendas comunitarias, que constituyen una estrategia de cadenas de comercialización directa. 


\section{Elementos de la Agricultura Familiar Campesina}

La agricultura familiar campesina, está caracterizada por diferentes tipologías. Estas buscan mostrar la unión de la familia mediante la entrega de un universo rural, dando a conocer las diferentes maneras de agricultura y las diversas actividades que se realizan. Esto se consigue a través de la participación de las familias (Carmagnani, 2008).

La Agricultura familiar, se caracteriza por destacar dos elementos fundamentales: el trabajo de la familia y su relación con la producción, es decir, la relación entre familia y el aprovechamiento agrícola. Para algunos analistas de la agricultura familiar en países del primer mundo (algunos países de Europa), esta es la relación clave que se debe considerar y sobre todo mirar cómo ha evolucionado en el medio rural (Mundler, Patrick et Rémy, Jacques 2012; Hervieu, Bertrand et Purseigle, Francois, 2011). A partir de ello, se podría ver la posibilidad de elaborar algunas tipologías de agricultura familiar, con el fin de considerar principalmente los vínculos con el mercado.

El presente estudio considera la participación de los productores representantes de la agricultura familiar campesina quienes constituyen las cadenas agroalimentarias. Estas cadenas están integradas por redes de organizaciones que trabajan de manera conjunta en diferentes procesos y actividades con el fin de entregar productos o servicios al mercado y satisfacer las demandas de los clientes cuidando siempre la calidad.

La presente investigación plantea optimizar las cadenas de distribución a partir de la identificación y caracterización de los principales obstáculos a lo largo de las rutas de la agricultura familiar campesina. Se identifican los aspectos importantes referentes a los obstáculos que tienen las organizaciones para poder llegar a mercados adecuados y diferenciados donde valoren los productos de calidad.

El principal objetivo es el de diseñar un modelo de optimización de la cadena de distribución de la agricultura familiar campesina, para el caso de las parroquias Quingeo y Santa Ana del cantón Cuenca. Al momento, no existen documentos que se hayan elaborado sistemática y rigurosamente para conocer los problemas que tienen las organizaciones y sobre las condiciones en las que sacan sus productos para la venta final.

\section{Metodologia.}

\section{Diseño de la investigación}

El método propuesto para el estudio se basó en un esquema descriptivo y transversal. La parte descriptiva se utiliza para caracterizar la cadena de distribución y estructurar los componentes de un modelo de optimización de las organizaciones en estudio. En primer lugar, se establece una caracterización y exploración de las variables en función de su naturaleza esencialmente del tipo categóricas. Se aplicaron métodos inferenciales sobre 
todo para respaldar los resultados mediante pruebas estadísticas que permitan extraer conclusiones válidas para conformar el modelo propuesto. El carácter transversal de este estudio responde a que los datos se recolectaron en un solo instrumento administrado y en un único momento en el tiempo.

\section{Población y muestra}

Se partió de un grupo constituido por 13 asaciones correspondientes a las parroquias de Quingeo y Santa Ana del Cantón Cuenca, dando un total de 182 productores asociados, a quienes se realizaron las encuestas. Para tener una mejor comprensión, el cuestionario fue agrupado por temas.

Para el estudio se aplicó el cuestionario a los 182 productores. El mayor porcentaje de agricultores corresponde a la Parroquia Quingeo con el $79 \%$, y Santa Ana en menor proporción con el $21 \%$. Es importante resaltar que el $70 \%$ de los agricultores pertenece al género femenino y se dedica a realizar las labores de labranza de sus tierras. Con respecto a la instrucción el mayor porcentaje corresponde a primaria con el $86 \%$, secundaria con el $9 \%$, educación superior con el $4 \%$ y por último el $2 \%$ corresponde solo a mujeres que no tienen ninguna formación.

\section{Instrumento de recolección de datos}

La investigación se llevó a cabo mediante encuestas y el instrumento de recolección de datos fue mediante un cuestionario de 43 ítems. El mismo estuvo conformado por dos partes, el primero corresponde a una sección informativa que recopila datos personales tales como género, edad, instrucción, organización a la que pertenece, tiempo de trabajo como organización, y parroquia de pertenencia (ítems 1-10). El segundo incluye cuestionamientos dirigidos a poder caracterizar la cadena de distribución de la agricultura familiar campesina para el caso de Quingeo y Santa Ana (ítems 11-43).

La caracterización de la cadena de distribución se efectuó aplicando un cuestionario adaptado a los problemas o dificultades que tienen los pequeños productores al momento de distribuir o vender sus productos, lo que permite identificar los canales de distribución que actualmente tienen y poder crear un modelo que sirva como instrumento para los pequeños y medianos productores.

El instrumento utilizado para la recolección de la información fue estructurado y aplicado vía online mediante una herramienta formulada en Google Forms. Para ello se realizaron llamadas telefónicas debido a que la mayoría de los productores no cuentan con correo electrónico. Es importante anotar que la información recopilada es de carácter anónimo y estuvo orientada a medir las dificultades que existen dentro de la cadena de distribución. La información recolectada mediante las encuestas fue organizada en una hoja electrónica (Excel) para luego procesarse mediante el programa de análisis estadístico Infostat (versión 2020). Los datos fueron de carácter categórico y por lo tanto su análisis se realizó 
mediante pruebas de $X^{2}$ (chi cuadrado), mismas que se emplearon para verificar la independencia de las variables consideradas en las tablas de contingencia. En las tablas de contingencia se colocaron los datos de la encuesta organizados en filas y columnas de acuerdo a las variables de interés, registrando los totales (frecuencias) de acuerdo a cada una de las categorías de las preguntas. La información de las tablas de contingencia fue representada en gráficos de barras para posteriormente, con aquellas relaciones más significativas, realizar un análisis multivariado para datos categóricos mediante Análisis de Correspondencias. Esto permitió, además, establecer que variables fueron más relevantes en el proceso y descartar otras o reconocer su escasa relevancia. Los resultados de este Análisis de Correspondencias fueron utilizados para elaborar un modelo de optimización de la cadena de distribución.

\section{Resultados.}

Los datos fueron analizados de acuerdo a varias dimensiones, donde cada una de estas consta de un número determinado de ítems vínculos al tema de la cadena de distribución de la agricultura familiar campesina para el caso de las parroquias Quingeo y Santa Ana (Tabla 1).

Para la presentación e interpretación de los resultados se realizó un tipo de análisis descriptivo, en este análisis se realizó un diagnóstico general de la cadena de distribución para conocer: a) el grado de dificultades que tienen los agricultores en la distribución de sus productos y b) en qué aspectos se desenvuelven con mayor facilidad y detectar el nivel de problemas que tienen.

Tabla 1: Dimensiones utilizadas para el análisis

\begin{tabular}{llc}
\hline \multicolumn{1}{c}{ Tema } & \multicolumn{1}{c}{ Dimensión } & ítems \\
\hline Datos personales & Instrucción & 4 \\
& Genero & 2 \\
& Asociación & 13 \\
& Parroquia & 2 \\
& Nivel de participación en la & 3 \\
& comercialización & \\
\hline $\begin{array}{l}\text { Distribución } \\
\text { los } \\
\text { productos }\end{array}$ & Tipo de producción & 3 \\
& Apelativos & 5 \\
& Materiales para transportar & 7 \\
& Costos de transporte & 5 \\
\hline
\end{tabular}




$\begin{array}{ll}\text { Lugar de comercialización } & 5 \\ \text { Falta de promoción } & 3 \\ \text { Canales de distribución } & 5 \\ \text { Calidad del producto } & 3 \\ \text { Dificultades de distribución } & 5\end{array}$

\section{Análisis descriptivo}

A continuación, se presenta los resultados de las dificultades de la cadena de distribución de la agricultura familiar campesina.

La dificultad de la cadena de distribución se concibe en base a la vulnerabilidad económica de los agricultores (Rebaï, 2017), ya que la falta de acceso al mercado, debido a su innegable subordinación a actores intermediarios que limitan su integración comercial, provoca la ocurrencia de monopolios, precios injustos, etc. Esto constituye un objetivo primordial, para la construcción de un modelo que sirva como herramienta que permita al agricultor mejorar su situación actual.

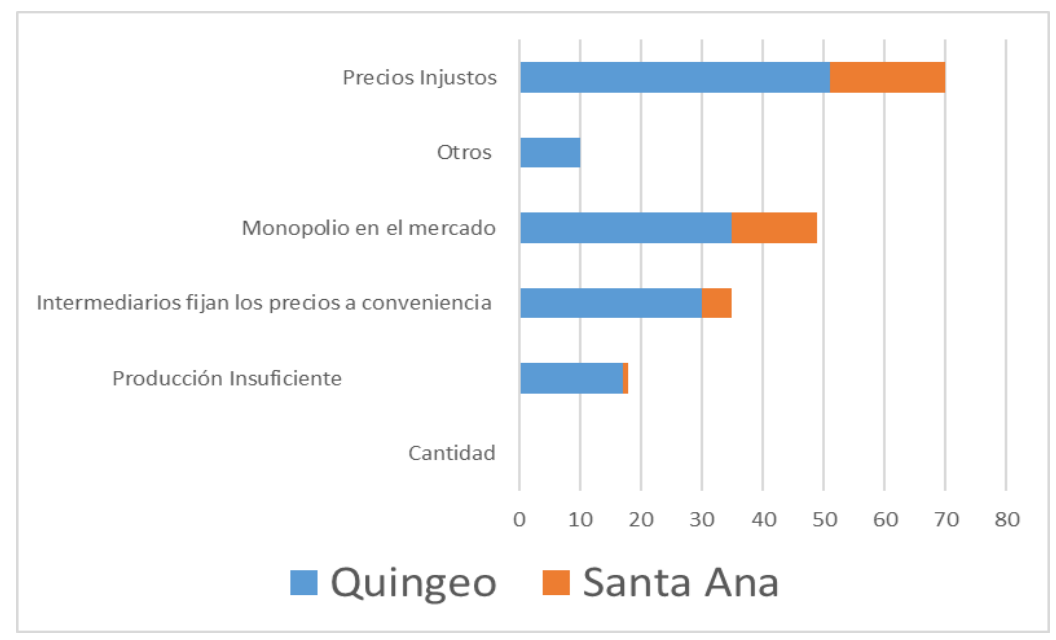

Figura 2: Dificultades en la cadena Distribución Fuente: Elaboración propia.

Uno de los elementos analizados en este apartado muestra que de los agricultores familiares campesinos en Quingeo, el $51 \%$ coinciden en que obtienen precios injustos (19\% en Santa Ana) y que sería importante aplicar una estrategia para evitar que se continúe dando esta situación, mientras que el $35 \%$ de estos siente un descontento (14\% en Santa Ana) debido al monopolio en los mercados (Figura 2). El $30 \%$ está en desacuerdo con que los intermediaros fijen los precios de los productos a conveniencia de ellos y que además se sume la falta de difusión (5 \% en Santa Ana). 


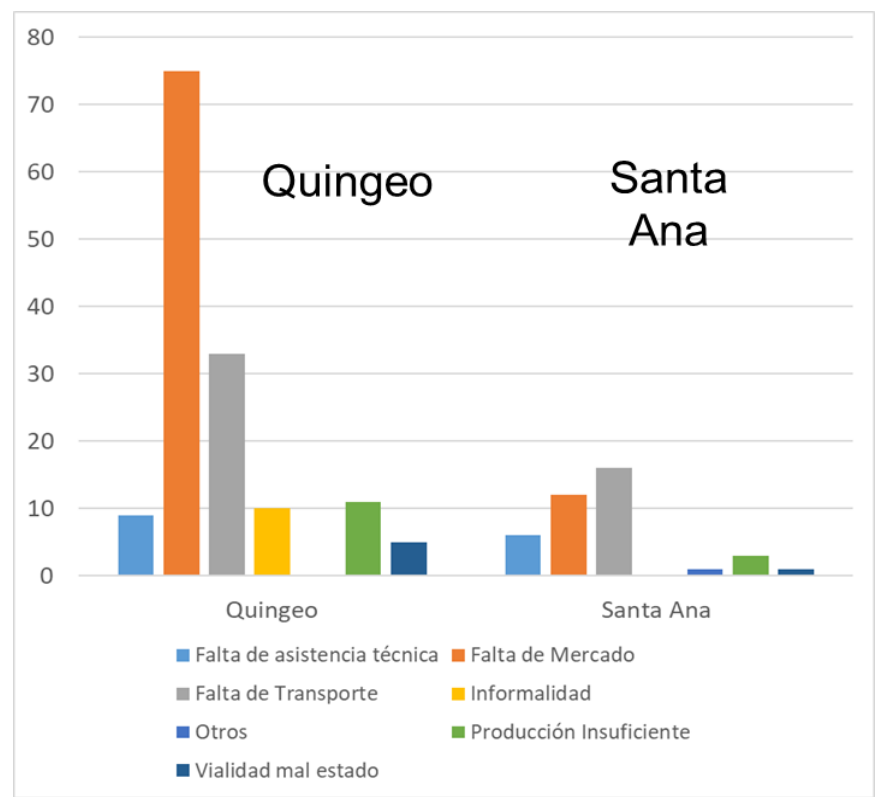

Figura 3: Dificultades en la cadena Distribución Fuente: Elaboración propia.

De los resultados en cuanto a dificultades en la cadena de distribución para los productores de Quingeo y Santa Ana, como se muestra en la (Figura 3), se identifica claramente que las dificultades más frecuentes para Quingeo son: la falta de mercados con el $75 \%$ y la falta de transporte con el $33 \%$, y en menor cantidad la producción insuficiente con el $11 \%$, la falta de asistencia técnica con el $9 \%$ y por último, pero no menos importante la vialidad en mal estado con el $5 \%$. En Santa Ana las dificultades son: falta de transporte con el $16 \%$, Falta de mercado con el $12 \%$, falta de asistencia técnica con el $6 \%$, producción insuficiente $3 \%$ y en mínima cantidad otros y vialidad en mal estado. En función de esto manifiestan que estarían de acuerdo y se comprometerían en aplicar el modelo que se les recomiende para mejor esta situación.

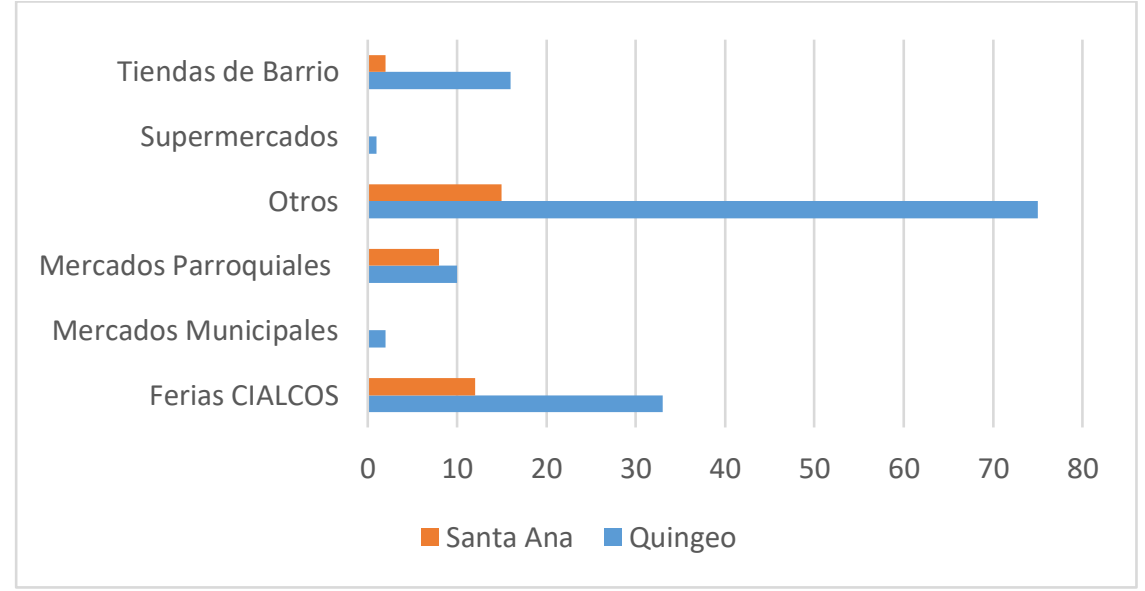

Figura 4: Lugares de Comercialización Fuente: Elaboración propia.

La dimensión que representa la comercialización es evaluada mediante seis ítems, y los resultados obtenidos muestran que la categoría Otros tiene el 75\%, donde los agricultores manifiestan que venden al intermediario productos tales como leche, cuy faenado y pollos 
(Figura 4). Adicionalmente con el apoyo del GAD Quingeo realizan canastas para ser entregadas a domicilio. El 33\% afirma que expenden sus productos en ferias de Circuitos Alternativos de Comercialización (CIALCOS). El 18\% vende en las ferias de barrio, el $10 \%$ en marcados parroquiales, el $3 \%$ venden en marcados municipales y por último $1 \%$ venden en los supermercados (para este último solamente los productores de Quingeo). Se evidencia el descontento de los agricultores por los precios injustos, donde el intermediario compra de acuerdo a la conveniencia del momento. Con la creación de las ferias CIALCOS hay un nexo directo entre productor y consumidor, alcanzando el segundo y tercer lugar en porcentaje para Quingeo y Santa Ana respectivamente (Figura 5). Esto concuerda con Barrionuevo (2018) quien aplicó ideas de impulsar estrategias de comercialización alternativa de alimentos provenientes de los pequeños y medianos productores a través de CIALCOS.

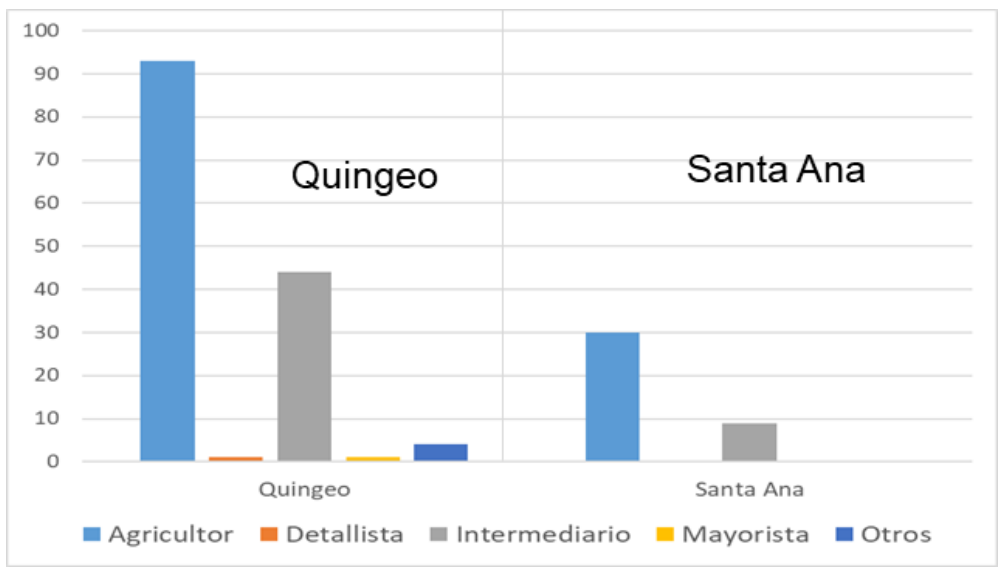

Figura 5: Canales de Distribución Fuente: Elaboración propia.

Con respecto a Canales de Distribución, como se ve en la (Figura 5), se observan claramente dos canales bien identificados: a) el directo donde hay una relación directa entre productor y consumidor final reflejado por el porcentaje más alto que corresponde a Agricultor (93\% y 30\% respectivamente), y b) indirecto caracterizándose por la relación: productor - intermediario - consumidor final (basado en que el 2do canal en importancia es Intermediario.

Los resultados de las encuestas correspondientes al Control de la Calidad de los productos donde se identifican 4 variables significativas tales como: a) sabor, tamaño, frescura, b) sabor y frescura, c) Sabor color y frescura y d) sabor; evidenciando que el agricultor si puede identificar la calidad de los productos que ofertan en el mercado.

Con respecto a las labores de Pos-cosecha, se identifican dos variables importantes tales como: a) clasifica y b) lavan los productos, con la finalidad de ofrecer calidad y presentación hacia el consumidor final.

Los medios de transporte identificados corresponden a cuatro categorías que serían las más importantes empleadas para llevar los productos hacia el mercado. El factor con mayor porcentaje es a) Otros y que corresponde a la utilización de tanques para almacenar 
y transportar la leche y coolers para almacenar los productos cárnicos, el siguiente es b) gavetas, c) gavetas otros y por ultimo d) canastas.

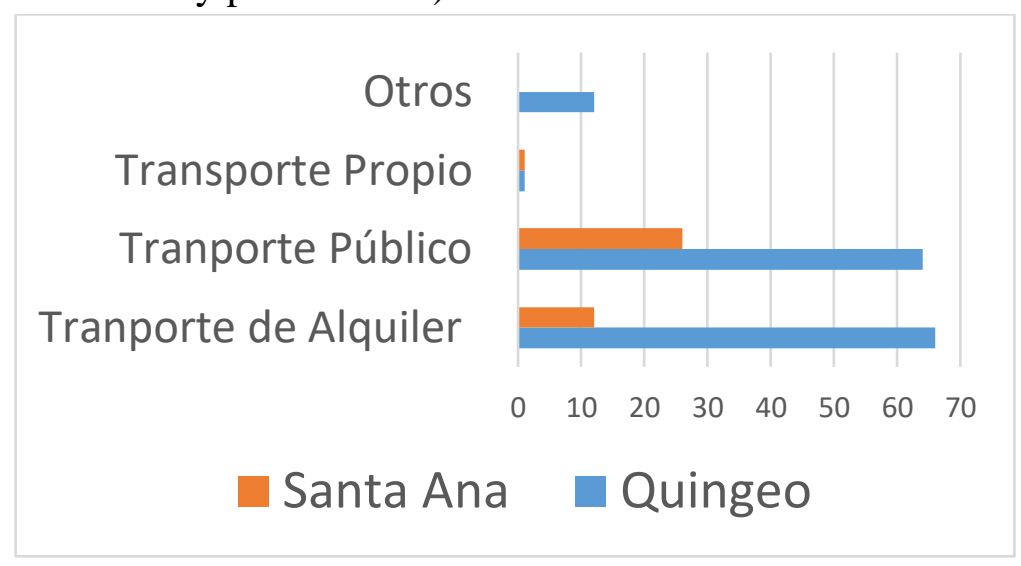

Figura 6: Uso de Transporte Fuente: Elaboración propia.

Referente al Uso de transporte, se evidencia que existen cuatro medios principales para transportar los productos (Figura 6), los dos con el mayor porcentaje y utilizados por los productores son: Transporte de Alquiler y Transporte público, y en menor proporción Otros. El medio menos utilizado corresponde a Transporte propio (1\% para las dos parroquias), razón por la cual muchos agricultores, manifestaron que durante la pandemia tuvieron pérdidas debido a la interrupción del servicio de transporte público. Por esto se vieron forzados a utilizar más el transporte de alquiler e incurrir en altos costos por hacer uso de este rubro y que en consecuencia sus ganancias fueran mínimas.

Existen tres factores importantes con respecto a los Problemas de las ventas: el primero con un alto porcentaje es la Calidad y el Precio del producto, el segundo y tercero con un mismo porcentaje corresponde a que los cuyes no cumplen con el peso que solicita el mercado y que el precio no es el adecuado, lo que conduce a que el agricultor obtenga pérdidas y una muy baja rentabilidad.

Con respecto a los Días de Ventas, se tienen tres categorías, a primera corresponde a que sus ventas las realizan diariamente, la segunda corresponde a ventas semanales, y la tercera a ventas quincenales. Por último, aunque con el menor porcentaje, es importante mencionar que hay productores que salen a expender sus productos de manera mensual. En lo que respecta al Costo para Transportar los Productos, lo que causa más gastos al agricultor es la mano de obra, seguido por el alquiler del vehículo, y en menor porcentaje otros y empaque. 


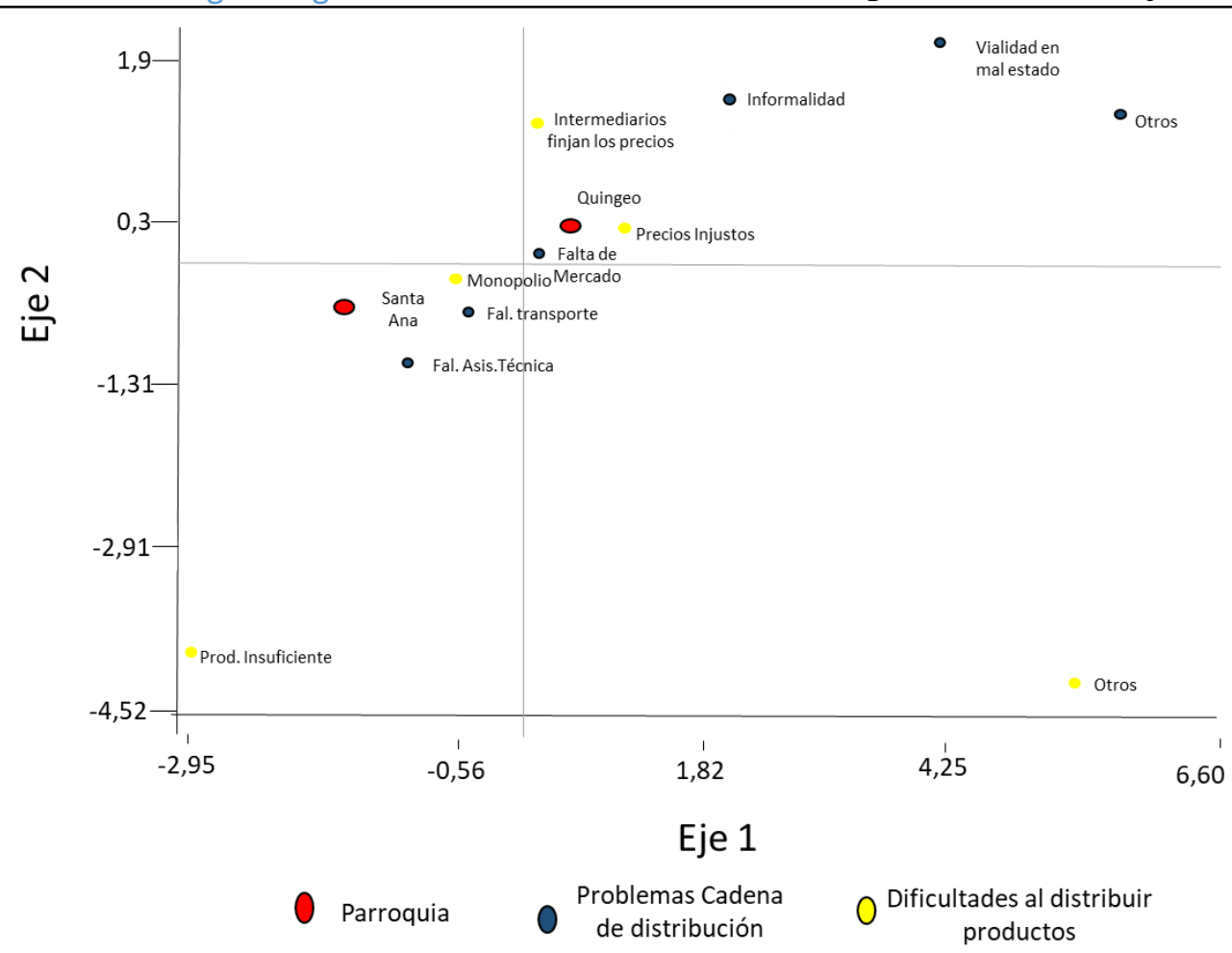

Figura 7: Análisis de Correspondencias para identificar la relación entre las variables de: a) Problemas de la Cadena de Distribución y b) Dificultad al Distribuir los productos Fuente: Elaboración propia.

A partir de las relaciones más significativas obtenidas mediante las tablas de contingencia se realizaron análisis de correspondencias como el que se presenta en la (Figura 7), donde se observa que hay una relación fuerte entre los factores de la variable correspondiente a las dificultades de distribución de productos tales como: la Falta de mercados, Precios injustos y los Intermediarios quienes fijan los precios a conveniencia de ellos. Estos factores al estar más cercanos presentarían un mayor impacto negativo debido a los efectos sinérgicos. En otro grupo, aunque más dispersos y por lo tanto menos relacionados entre sí, estarían problemas de distribución tales como: Informalidad y Vialidad en mal estado y Otros. Por otro lado, se evidencia que hay una correspondencia entre: la Falta de transporte, Monopolios, y la Falta de asistencia técnica. La Producción insuficiente y Otros aparece más bien como factores aislados. Esto evidencia que el principal problema en las dos parroquias es la Falta de mercados y Transporte, constituyendo un factor de riesgo bastante considerable para los productores de la Agricultura Familiar Campesina.

Propuesta de modelo de cadena de distribución de la agricultura familiar campesina Con el propósito de estructurar un modelo de optimización de la cadena de distribución de la agricultura familiar campesina, el estudio se basó en el diagnóstico y el análisis de los resultados obtenidos a partir de los datos que se levantaron mediante las encuestas realizadas a los agricultores. El propósito de este modelo es el de brindar una herramienta que pueda ser aplicada por los productores y que por lo tanto les sirva de apoyo para conseguir mejorar sus ventas y satisfacer con sus productos las expectativas de los consumidores. 


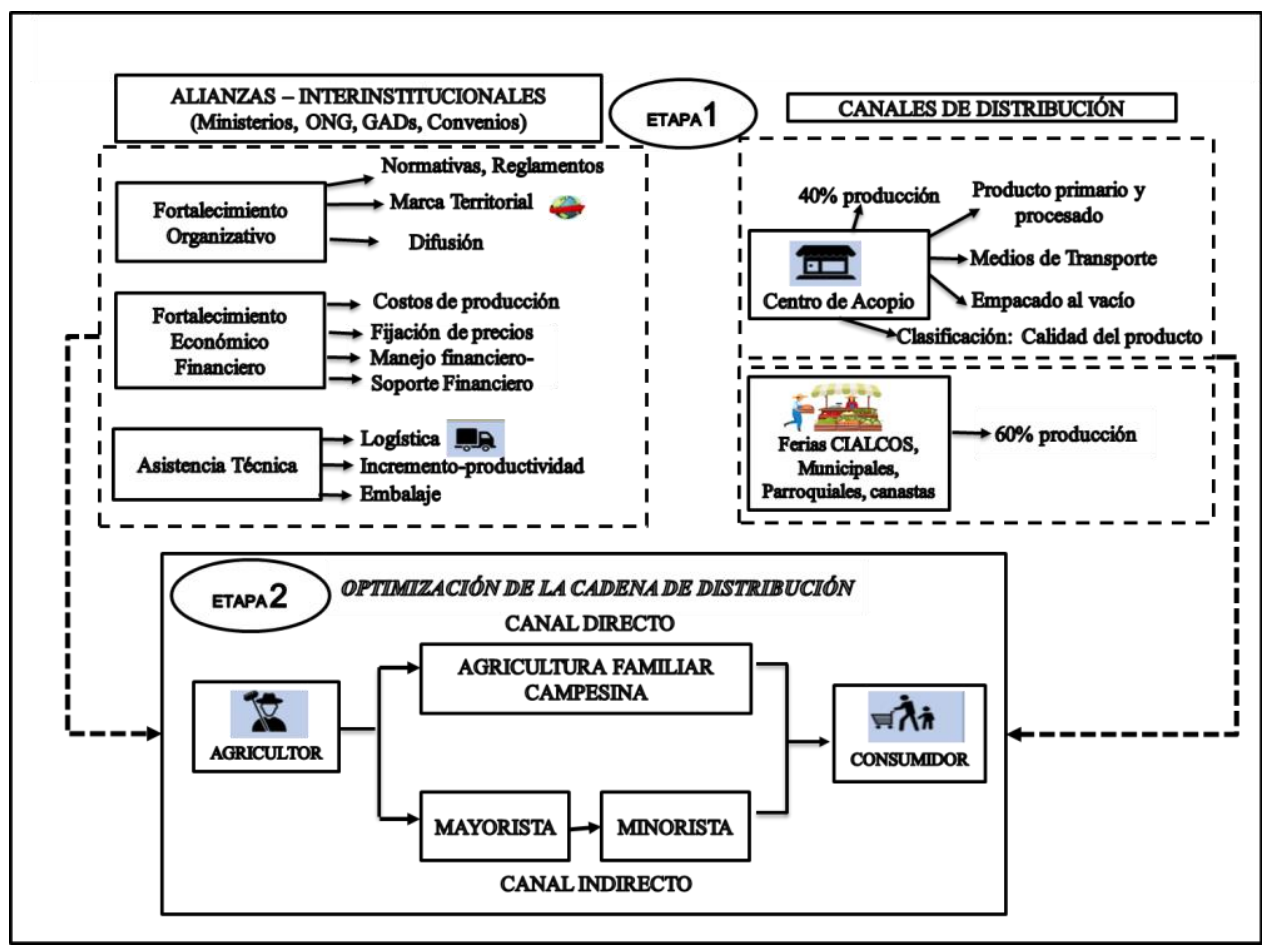

Figura 8: Modelo de optimización de la cadena de distribución de la agricultura familiar campesina

Fuente: Elaboración propia.

En la (Figura 8) se puede observar el modelo planteado a partir de los resultados de la investigación, que consiste en la implementación de dos etapas. En la primera etapa tenemos cuatro estrategias tales como: Alianzas, Fortalecimiento a varios niveles y Asistencia técnica.

Dentro de la segunda etapa constan dos Canales de Distribución: a) Directo y b) Indirecto.

La primera etapa consiste en establecer Alianzas interinstitucionales con el fin de generar proyectos y convenios en temas productivos y comerciales. Para ello se considera estratégico fortalecer a la organización mediante la creación de normas y reglamentos que permitan una adecuada estructuración a nivel de organización, con una madurez que les ayude a trabajar y comercializar asociativamente. En este sentido, es indispensable crear en una marca territorial que cobije a todas las organizaciones tanto de Quingeo como Santa Ana que les distinga de otras organizaciones y que les permita ser competitivas al momento de difundir sus productos mediante redes sociales, televisión, prensa escrita, etc.

Con respecto al Fortalecimiento Económico Financiero, es importante que las organizaciones conozcan cómo elaborar y reducir los costos de producción, para ser competitivos en el mercado, tener conocimiento y manejar precios de acuerdo al mercado, y constantemente capacitarse en manejo financiero. 
Referente a la asistencia técnica, con la tecnificación de los cultivos, mejoran la productividad, tienen variedad de productos, realizan adecuadamente las labores de cosecha y pos cosecha obteniendo un producto de calidad.

Dentro de la primera etapa también constan otras actividades de soporte correspondientes a los Canales de Distribución. Aquí se implementa el Centro de acopio, mismo que permite facilitar las labores de pos cosecha y comercialización, además ayudaría a los agricultores a vender su producción sobrante del $40 \%$. Aquí también se pueden ubicar los productos primarios y los procesados.

Con la finalidad de que los productos se mantengan frescos es indispensable utilizar medios de transporte adecuados tales como: gavetas, cartones, canastas, coolers, etc. y complementar con un adecuado

Por último, en esta etapa, se tiene a las Ferias CIALCOS, en donde los agricultores venden el $60 \%$ de su producción. En este espacio no hay intermediarios y hay una conexión directa entre productor y consumidor final.

La Segunda Etapa contempla La Optimización de la Cadena de Distribución, para lo cual importante cumplir con las actividades de soporte de la primera etapa, como se puede observar en la (Figura 8), en donde se observan dos canales de distribución:

1) Canal Directo: Aquí tenemos al Productor de la Agricultura Familiar Campesina y al consumidor final, entre quienes existe un nexo directo al eliminarse el intermediario. El total de ventas a través de este canal es del $60 \%$.

2) Canal Indirecto: Aquí intervienen el Productor, el Mayorista (quien posee el centro de acopio), el Minorista y Consumidor Final. El total de ventas a través de este canal es del $40 \%$. De esta forma, mediante los dos canales, los agricultores logran comercializar el $100 \%$ de su producción, evitando perdidas y dinamizando la economía local.

\section{Conclusiones.}

- Este artículo presenta una revisión de las principales teorías acerca del desarrollo económico local y de las cadenas de distribución relacionadas con la agricultura familiar campesina, evidenciando que, si bien existen muchos trabajos e iniciativas al respecto en el país, estos trabajos no han sido publicados por lo que la literatura científica es más bien escasa. Sin embargo, esta revisión dio paso a la identificación de los aspectos más relevantes involucrados en la temática y permitió la elaboración de una estrategia para definir un modelo a partir de los datos generados en este estudio. Además, dejó en evidencia lo complejo de las relaciones entre los elementos que conforman una red de distribución.

- En cuanto a las amenazas a nivel de las cadenas de distribución, el estudio mostró que los factores de riesgo más importantes son la falta de mercados, precios 
injustos, transporte, falta de asistencia técnica y monopolio a nivel de los mercados. Muchas veces estos factores pueden estar actuando solos o en conjunto en cuyo caso su efecto se ve potenciado por la interacción que ejercen.

- Con respecto a los factores que están relacionados con los problemas de distribución de los productos, el análisis descriptivo permitió evidenciar dos tipos predominantes de canales. El primer canal es de tipo directo y se caracteriza porque el productor tiene contacto con el consumidor. Por otro lado, el segundo canal es de tipo indirecto y aquí aparece el intermediario como vínculo entre el productor y el consumidor.

- A partir de un análisis de las relaciones que se dan entre las variables involucradas y agrupadas de acuerdo a las dimensiones de la Distribución de los productos y de sus correspondientes ítems, fue posible definir un modelo de optimización de la cadena de distribución que visualiza la complejidad del proceso y que pone de manifiesto cuáles son los aspectos en donde se tiene que poner mayor énfasis para conseguir potenciar los puntos débiles a lo largo del proceso. Sin bien los problemas son recurrentes y evidentes en ambas parroquias, en algunos casos la forma en la que interactúan y se manifiestan no es la misma, por lo que los modelos obtenidos para una determinada zona podrían ser aplicables en otros lugares solamente después de haber sido validados.

- Finalmente, para futuras investigaciones sería conveniente realizar un diagnóstico de la cadena de distribución involucrando más parroquias de modo que el modelo pueda ser de carácter más global.

\section{Referencias bibliográficas.}

Ayora, D. (2016). Repositorio Instituto de Altos Estudios Nacionales. Obtenido de La pluriactividad como proceso de descampenización juvenil en las Unidades Domésticas Campesinas Caso Parroquia El Valle.

Ahi, P., \& Searcy, C. (2013). A comparative literature analysis of definitions for green and sustainable supply chain management. Journal of Cleaner Production, 52, 329341.

Barrionuevo, N., Chehab, C., Andrade, D., \& Morillo, J. (2018). Identificación y caracterización de mecanismos de articulación de la oferta de la agricultura familiar a mercados. 45 .

Bingham y Mier, (1993). Teorías del Desarrollo Económico Local.

Cartier. (2016). Apuntes de catedra: Costos para la Gestión.

Carmagnani. (2008). La agricultura familiar en América Latina.

Clark, G. Huxley, J., \& Mountford, D. (2012). La economía local: la función de las agencias de desarrollo. In Serie Políticas Públicas y Transformación Productiva $\mathrm{N}^{\circ}$ 
2.

Díaz, Campos, Gómez. (2012), Cadena Agroalimentaria en España.

Fundación Heifer Internacional. (2018 ). Obtenido de Agricultura Familiar Agroecológica.

Hervieu, Bertrand et Purseigle, Francois. (2011). "Des agricultures avec des agriculteurs, une nécessité pour l'Europe.

Kirwan, J., Maye, D., \& Brunori, G. (2017). Acknowledging complexity in food supply chains when assessing their performance and sustainability. Journal of Rural Studies Journal.

Maletta, H. (2016). Tendencias y perspectivas de la agricultura familiar.

Manrique. (2011). Manual de cadenas de valor.

Morelos Gómez, J. (2012). Análisis de la Cadena de Suministro de un Hipermercado. Ingeniare, 13, 11.

Mundler, Patrick et Rémy, Jacques (2012).'L'exploitation familiale à la francaise. : une institution dépassé ?”, en , L’Homme et la Société.

Naciones Unidas. (2016). Las cadenas de valor de los productos básicos agrícolas: los efectos de la concentración del mercado para los agricultores y los países productores - El caso del cacao. 17838, 17.

Neven, D. 2009. Three steps in value chain analysis. Micronota n. ${ }^{\circ}$ 53. Washington, D.C., Agencia de los Estados Unidos para el Desarrollo Internacional.

Lechón , W., \& Chicaiza , J. (2019). Eutopia. Revista de Desarrollo Económico Territorial N. ${ }^{\circ} 15$, junio 2019, pp. 193-210. Obtenido de De la agricultura familiar campesina a las microempresas de monocultivo. Reestructura socioterritorial en la sierra norte del Ecuador.

Orellana. (2001) Midiendo la pobreza y la privación en Ecuador.

Organización de las Naciones Unidas para la Alimentación y la Agricultura . (2014). Obtenido de http://www.fao.org/3/a-as281s.pdf

Plan Nacional del Buen Vivir . (2017). Obtenido de https://www.gobiernoelectronico.gob.ec/wpcontent/uploads/downloads/2017/09/Plan-Nacional-para-el-Buen-Vivir-20172021.pdf

Peñafiel, L. (2017). Aproximación de la agricultura familiar campesina por el método 
análisis multiescala integrado de los metabolismos sociales .

Pérez. (2004) Liberación financiera, crisis y destrucción de la moneda nacional en Ecuador.

Pino , L., Hermes, R., Apolo , G., \& Sisalema, L. (2018). Revista Espacios ISSN 0798 1015. Obtenido de Aporte del sector agropecuario a la economía del Ecuador: http://www.revistaespacios.com/a18v39n32/a18v39n32p07.pdf.

Porter, M. (1986). Ventaja Competitiva. Editorial C.E.C.S.A. México.

Secretaría Nacional de Planificación y Desarrollo, (2017-2021). Toda una vida.

Reefke, H., \& Sundaram, D. (2016). Key themes and research opportunities in sustainable supply chain management identification and evaluation.

Rebaï, (2017). Vulnerabilidad de la agricultura familiar y de los territorios rurales en los Andes ecuatorianos. Un análisis desde la provincial del Azuay.

Vázquez Barquero, A. (2000). Desarrollo económico local y descentralización: Aproximación a un marco conceptual. Revista de La Comisión Económica Para América Latina y El Caribe (CEPAL), 1-50.

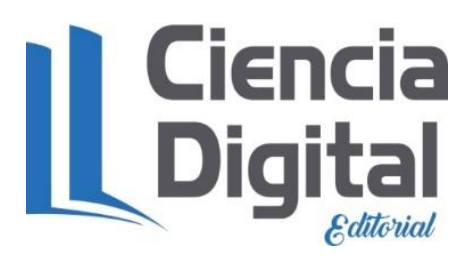




\section{PARA CITAR EL ARTÍCULO INDEXADO.}

Piedra Rivas, M. C., Banegas Campoverde, C. M., \& Castillo Ortega, Y. (2021). Modelo de optimización de la cadena de distribución de la agricultura familiar campesina en las parroquias Quingeo y Santa Ana del Cantón Cuenca . ConcienciaDigital, 4(2), 114-134. https://doi.org/10.33262/concienciadigital.v4i2.1630

\section{Liencia}

El artículo que se publica es de exclusiva responsabilidad de los autores y no necesariamente reflejan el pensamiento de la Revista Conciencia Digital.

El artículo queda en propiedad de la revista y, por tanto, su publicación parcial y/o total en otro medio tiene que ser autorizado por el director de la Revista Conciencia Digital.

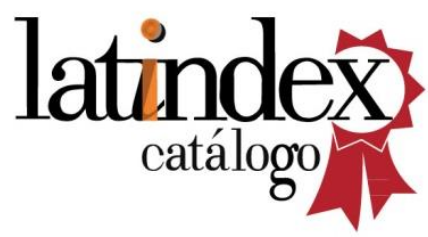

